\title{
Spontaneous external biliary fistula uncomplicated by gallstones
}

\author{
B.R.P. Birch and S.J. Cox \\ Department of Surgery, Watford General Hospital, Vicarage Road, Watford, Hertfordshire, UK
}

Summary: External biliary fistulae are rare. Only 65 cases have been reported in the literature and in each instance gallstones were a complicating factor. We report in this paper the first case of spontaneous external (cholecystocutaneous) biliary fistula uncomplicated by gallstones.

\section{Introduction}

External biliary fistulae, first described by Thilesus in 1670 and common in the last century, have become rare since the advent of modern biliary surgery. There have been just 65 cases recorded since $1900^{1,2}$ and all of these were complicated by gallstones. We report here the first case of spontaneous external biliary fistula in which gallstones were not a complicating factor.

\section{Case report}

A 79 year old woman was admitted with a painful mass in the right upper quadrant of her abdominal wall. She gave a history of having noted a painful swelling on the right side of her abdomen, which had slowly enlarged over a 2-week period. This burst spontaneously just after admission, with subsequent relief of her pain. No gallstones were discharged at this or any subsequent time. A foul-smelling, purulent discharge persisted.

On examination, she was noted to be pale and obese and had a low grade pyrexia. The abdomen was soft with a necrotic area in the right upper quadrant discharging foul-smelling, purulent material from several sinuses. No masses could be distinguished with certainty and rectal examination was normal.

Initial investigations showed her to be anaemic with an haemoglobin of $8.4 \mathrm{~g} / \mathrm{dl}$ and white cell count $8.4 \times 10 / 1$ ( $84 \%$ neutrophils). A plain abdominal radiograph showed surgical emphysema of the soft tissue of the right abdominal wall. All blood tests were otherwise normal and swabs taken for culture grew coliforms with Bacteroides fragilis.

Correspondence and present address: B.R.P. Birch, M.A., F.R.C.S., Institute of Urology, 172 Shaftesbury Avenue, London WC2, UK

Accepted: 2 May 1990
The necrotic area of the abdominal wall was initially debrided under local anaesthesia with antibiotic cover. The patient subsequently became apyrexial and was transfused to correct her anaemia.

Four days after debridement the patient was returned to theatre for examination under anaesthetic. This showed there was a very narrow fistula communicating intra-abdominally. A decision was made to proceed to laparotomy. An incision was made encompassing all necrotic tissues on the abdominal wall and the fistula was seen to be communicating with the fundus of the gallbladder, which was adherent to the anterior abdominal wall. The gallbladder did not contain any stones, the common bile duct was of normal calibre with no palpable calculi, and the pancreas was normal.

The gallbladder and fistula were excised en bloc and the defect in the abdominal wall closed primarily in layers. Drains were left to the gallbladder bed and to the abdominal wound. Histology of the removed gallbladder showed severe active chronic inflammation and granulation tissue. There was no evidence of malignancy. Postoperatively the patient made an uneventful recovery. Outpatient review at 6 and 12 weeks showed her to be well with a soundly healed abdominal wound.

\section{Discussion}

Niemeier ${ }^{3}$ classified perforation of the gallbladder into acute, subacute and chronic varieties, only the latter being characterized by fistula formation. Of such fistulae the majority are enteric ${ }^{4}$ (duodenum $77 \%$, colon $15 \%$ ) while external fistulae are rare.

External biliary fistula formation is chiefly a disease of females in the 5 th -7 th decades, reflecting the increased incidence of cholecystitis in this age group. The majority of these fistulae open 
into the right upper quadrant $(48 \%)$ or umbilicus $(27 \%){ }^{1}$

Perforation of the gallbladder without stones is said to complicate $0.6-1 \%$ of all cases of acute cholecystitis. ${ }^{5}$ The aetiology of perforation in such cases is not clear, although bacteraemia, steroids, polyarteritis nodosa, typhoid and trauma have all been implicated. ${ }^{5}$ Roslyn and Busuttil ${ }^{6}$ have drawn attention to the importance of the roles of infection and circulatory changes in the pathogenesis of perforation in the elderly.

The net effect of such factors is to provide the necessary conditions for necrosis and perforation of the relatively avascular fundus. Prior in-

\section{References}

1. Henry, C.I. \& Orr, T.G. Spontaneous external biliary fistulae. Surgery 1949, 26: 641-646.

2. Fitchett, C.W. Spontaneous external biliary fistulae. Va Med 1970, 97: 538-543.

3. Niemeier, O.W. Acute free perforation of the gallbladder. Ann Surg 1934, 99: 922-924.

4. Glenn, F., Reed, C. \& Grafe, W.R. Biliary enteric fistula. Surg Gynecol Obstet 1981, 153: 527-531. flammatory adhesion to the abdominal parietes then allows external fistula formation after perforation.

The treatment of acute fistula requires adequate drainage, antibiotics and general supportive measures. Once the purulent drainage subsides fistulography can be performed, followed at a suitable interval by elective cholecystectomy with excision of the fistula. However, as up to $20 \%$ of all external biliary fistulae heal spontaneously ${ }^{1}$ the elderly debilitated patient may be treated conservatively and surgery deferred depending on symptoms.

5. Sherlock, S. Diseases of the Liver and Biliary System. 7th edition. Blackwell, Oxford, 1985, pp. 517-518.

6. Roslyn, J. \& Busuttil, R.W. Perforation of the gallbladder a frequently mismanaged condition. Am J Surg 1979, 137: $307-312$. 